\title{
Fatores comuns determinantes de crises cambiais e financeiras
}

\author{
CRISTINA FÁTIMA MARTINS DE BESSA \\ TITO BELCHIOR SILVA MOREIRA \\ MAURÍCIO DE PAULA PINTO BARATA \\ FERNANDO ANTÔNIO RIBEIRO SOARES*
}

Determinative common factors of currency and financial crisis. This paper identifies and evaluates determinative common factors of currency and financial crisis in relation to 86 crises episodes between 1970-2004, based on factor analysis, cluster and discriminant analysis. One evidenced that the rise of the ratios of domestic credit, fiscal deficit and residents bank deposits to the GDP is inherent to the different types of crises classified for economic literature. It was also identified as common factors to these episodes some indicators that capture the excessive monetary expansion of the economies and that reflect the fall in international reserves, represented by M2/Reserves and Imports/Reserves ratios and also the total volume of international reserves.

Keywords: currency and financial crisis; factor analysis; discriminant analysis. JEL Classification: F31; F41.

\section{INTRODUÇÃO}

Observando-se a economia mundial nos últimos 40 anos, verifica-se a alta e crescente incidência de crises cambiais e financeiras, atingindo tanto economias maduras quanto países em desenvolvimento. Os estudos empíricos têm sido eficientes na verificação da vulnerabilidade dos países aos colapsos cambiais, apesar da dificuldade na previsão das ocorrências desses episódios. Considerando-se que uma

\footnotetext{
* Respectivamente, Mestre em Economia pela Universidade Católica de Brasília: crisbessa@terra.com. br, Universidade Católica de Brasília. E-mail: tito@pos.ucb.br. E-mail: Universidade de Brasília. E-mail: mauriciopinto@unb.br, Doutor em Economia pela UnB. E-mail: fernandosoares@unb.br. Submetido: Setembro 2007. Aprovado: Junho 2009.
} 
crise cambial é caracterizada pela perda repentina de confiança na moeda nacional e sua acelerada depreciação em relação a outras moedas, com reflexos no setor real da economia, percebe-se a importância da investigação dos ataques especulativos.

As teorias surgidas dessa investigação subdividem-se em três grupos de modelos: de primeira, segunda e terceira geração.

Os modelos de primeira geração tiveram como foco de estudo as crises na América Latina nos anos 1960 e 1970, relacionando as causas fiscais e monetárias às crises. De acordo com essa abordagem, as crises cambiais ocorreriam devido à contínua monetização do déficit fiscal, proporcional à perda de reservas, levando um regime de câmbio fixo ao colapso, pela exaustão dessas reservas.

A segunda geração de modelos voltou-se às causas das crises do Sistema Monetário Europeu em 1992, centrados na análise de economias maduras, que, mesmo com os fundamentos macroeconômicos sólidos, foram vítimas de ataque especulativo, provocando crises com caráter autorrealizável. Nestes casos, boatos não relacionados aos fundamentos monetários e fiscais tornam-se o centro das turbulências.

O terceiro grupo de análise foi consequência da onda de crises cambiais surgidas a partir da segunda metade da década de 1990: a chamada Crise Tequila em 1994 e, principalmente, as crises dos países asiáticos em 1997 e 1998 que se sucederam àquela crise, a moratória russa em 1998 e os colapsos brasileiro em 1999 e argentino em 2002. Moreira, Pinto e Souza (2004) apresentam uma revisão mais detalhada da literatura de crises cambiais e financeiras.

Este trabalho objetiva contribuir com a pesquisa empírica agregando a visão de fatores latentes que são comuns a crises de natureza diferentes, independentemente do estágio de amadurecimento da economia dos países envolvidos nos episódios ou da época de suas ocorrências.

Primeiramente, faz-se uma revisão da teoria que suporta a ligação entre pressão cambial e crises financeiras e das pesquisas empíricas relacionadas ao tema, incluindo os estudos voltados à sinalização e predição das turbulências. A seguir, estuda-se um conjunto de 20 países, num total de 86 ocorrências de crises, tanto de economias maduras quanto emergentes, no período de 1970 a 2004. Pretende-se, com a utilização de métodos estatísticos de análise multivariada, identificar e avaliar fatores comuns determinantes das crises cambiais e financeiras no período sob análise. Para esse fim, serão utilizadas técnicas de análise fatorial, análise de agrupamento e discriminante.

O artigo está constituído de cinco seções. Na segunda seção, faz-se uma breve revisão da literatura sobre indicadores de crises cambiais e financeiras. A terceira seção é dedicada à descrição da amostra estudada e à metodologia a ser utilizada na exploração e análise dos dados. Na quarta seção, discutem-se os resultados do trabalho e, por fim, na última seção, apresentam-se os comentários finais. 


\section{INDICADORES DE CRISES CAMBIAIS E FINANCEIRAS}

Um importante estudo sobre esse tema foi realizado por Eichengreen et al. (1996) para um grupo de 20 países da Organização Econômica de Cooperação e Desenvolvimento (OECD), utilizando observações trimestrais para o período 1959 a 1993. Frankel e Rose (1996) trabalharam com uma amostra de 105 países em desenvolvimento, usando observações anuais para o período 1971-1992.

Por sua vez, Kaminsky, Lizondo e Reinhart (1997) propõem uma metodologia específica para construir um sistema de aviso antecipado, que consiste em monitorar um conjunto de 15 variáveis macroeconômicas, cujo comportamento tende a ser incomum em momentos anteriores a crises. No caso de um determinado indicador ultrapassar certo limite, isso corresponderia a um sinal de que uma crise cambial poderá ocorrer nos próximos 24 meses. Se o sinal emitido é seguido pela ocorrência de uma crise em 24 meses, então o sinal é bom. Caso contrário, o sinal representa um ruído. Os resultados obtidos indicaram os melhores indicadores para antecipar crises: exportações, desvio da taxa de câmbio real em relação à sua tendência, razão M2/reservas e preço de ações.

Seguindo a literatura, este trabalho examina o perfil dos episódios de crise pela utilização de uma variedade de indicadores. A escolha dos indicadores buscou refletir os principais focos de estudo das várias gerações de modelos de crises cambiais. No total, são apurados 13 indicadores.

Os modelos de primeira geração ressaltam a inconsistência entre políticas macroeconômicas expansionistas e um regime de taxas de câmbio fixa. Nesse caso, déficits fiscais e políticas monetárias inconsistentes com a manutenção de câmbio fixo estão no centro dos modelos. Para capturar a essência desses modelos, são utilizadas as razões déficit fiscal/PIB, crédito doméstico/PIB e M2/Reservas (veja Apêndice).

No caso dos modelos de crises cambiais de segunda geração, o foco está no dilema do formulador de política econômica diante da relação custo-benefício da manutenção do regime de câmbio fixo. A manutenção do câmbio fixo tem consequências na liquidez da economia, nas taxas de juros, de inflação e desemprego, entre outras. Assim, dado que apreciações da taxa de câmbio real levam à perda de competitividade e recessão, são utilizados os mesmos indicadores sugeridos por Kaminsky (2003): as razões importações/reservas e exportações/reservas, taxa real de câmbio, termos de troca, índice de produção industrial e taxas reais de juros domésticas (veja Apêndice).

Para os modelos de terceira geração, a principal preocupação são os problemas financeiros que agravam as crises cambiais. Para essa classe de modelos, são utilizados indicadores que refletem as fragilidades referidas: razão entre crédito doméstico e PIB, a relação entre M2 e as reservas internacionais, a relação entre M2 e a base monetária, e a proporção de depósitos bancários de residentes em relação ao PIB (veja Apêndice).

Ressalte-se ainda que teorias de crises de inspiração minskiana podem for- 
necer subsídios interessantes para testes empíricos utilizando a mesma abordagem aqui empregada. ${ }^{1}$

\section{ASPECTOS METODOLÓGICOS}

Partindo dos resultados obtidos por Kaminsky (2003), em que foram identificadas seis variedades de crises cambiais e financeiras a partir do exame de episódios de crises em 20 países industrializados e em desenvolvimento, este trabalho tem por objetivo identificar os indicadores comuns aos diversos tipos de crises encontrados pela autora, por meio da utilização de técnicas estatísticas de análise multivariada.

Para permitir uma análise comparativa com o estudo realizado pela autora, são utilizados os mesmos eventos previamente classificados como episódios de crises, conforme índice de pressão no mercado de câmbio, ${ }^{2}$ totalizando 86 crises. Os episódios de crise para cada país da amostra estão relacionados em Kaminsky (2003).

Associado o cada episódio de crise, define-se um período considerado tranquilo, para verificar a existência de diferenças sistemáticas entre os dois períodos. O comportamento das variáveis nesse intervalo de tempo é examinado como a variação de seu valor na crise em relação ao período de tranqüilidade. São considerados períodos tranquilos os dois anos, anteriores à janela de tempo definida para o episódio de crise, garantindo assim que as observações fiquem fora da zona de turbulência. $\mathrm{Na}$ impossibilidade de considerar-se o período definido acima, em função da ocorrência sequencial de crises de um mesmo país, é escolhido o período imediatamente anterior que fica fora do intervalo de interferência dos eventos de crise.

Da mesma forma que nos eventos de crise, são considerados os mesmos 20 países do estudo original. O grupo de países desenvolvidos é composto por: Dinamarca, Finlândia, Noruega, Espanha e Suécia. O segundo segmento, formado por economias emergentes, engloba: Argentina, Bolívia, Brasil, Chile, Colômbia, Indonésia, Israel, Malásia, México, Peru, Filipinas, Tailândia, Turquia, Uruguai e Venezuela.

Nos moldes de Eichengreen et al. (1996), o estudo é desenvolvido a partir de observações trimestrais dos indicadores, compreendendo o período de janeiro de 1970 a dezembro de 2004. A disponibilidade dos dados também balizou a escolha da frequência das observações.

Em relação às variáveis macroeconômicas e financeiras do estudo, são utilizados 13 indicadores indicados pela literatura. A descrição dos indicadores e suas fontes estão relacionadas no Apêndice.

Para a análise dos dados e determinação dos fatores comuns determinantes

\footnotetext{
${ }^{1}$ Uma abordagem sobre crises financeiras com um enfoque à la Minsky pode ser visto em Nohara (1997).

${ }^{2}$ Para detalhamento da metodologia do índice de pressão sobre o mercado de câmbio, ver Kamisnky e Reinhart (1999).
} 
das crises é utilizado um conjunto de métodos estatísticos de análise multivariada. Esse conjunto engloba a análise fatorial, com associação da análise de componentes principais na extração dos fatores comuns, e, ainda, metodologias de análises de classificação, como análise discriminante e de agrupamentos. As técnicas de análise multivariada utilizadas neste estudo são descritas em Manly (2004) e Johnson e Wichern (1992).

As principais preocupações da literatura sobre crises cambiais e financeiras referem-se aos indicadores que podem prever tais crises e à identificação das diferentes naturezas ou causas das crises. Este artigo possui duas contribuições a essa literatura. Primeiramente, não estamos preocupados com o que diferencia cada episódio de crise ou com as diferentes naturezas das crises, mas com o que há em comum entre elas. Um estudo com esse enfoque ainda não tinha sido realizado. Essa é a primeira contribuição. Isto posto, precisaríamos de uma metodologia apropriada para investigar os fatores comuns relativo aos diferentes tipos de crises. Nesse contexto, temos uma segunda contribuição. Utilizamos inicialmente a análise fatorial para revelar os indicadores de crise comuns a todos os eventos analisados e, posteriormente, utilizamos a análise de classificação (análise de agrupamento e análise discriminante) para avaliar com base nos escores dos fatores comuns se há distinção de grupos de países por episódio de crise. Essa metodologia ainda não tinha sido empregada na literatura internacional.

\section{RESULTADOS E ANÁLISES}

O interesse deste trabalho é a identificação de um ou mais fatores comuns que determinam as crises cambiais e financeiras nos 86 episódios ocorridos no período entre 1970 e 2004, envolvendo tanto países desenvolvidos, como àqueles considerados emergentes.

Os indicadores macroeconômicos e financeiros aqui utilizados têm sido frequentemente aplicados em pesquisas empíricas por diversos autores, como Kaminsky e Reinhart (2003) e Frankel e Rose (1996), entre outros já citados, para identificar uma segmentação das crises em variedades diferentes e, ainda, na previsão dessas turbulências. Da mesma forma, os sinais esperados dessas variáveis são conhecidos a priori, conforme indica a literatura.

Pela utilização da técnica estatística de análise fatorial, que tem entre seus objetivos principais identificar o que há de comum entre os elementos de uma amostra sob estudo, espera-se também encontrar uma definição de grupos semelhantes com base nos fatores que determinam as crises, independentemente de suas diferentes naturezas diferentes.

A partir da identificação dos componentes (ou fatores) que permitem a redução dos 13 indicadores de crise a uma quantidade menor de fatores comuns, utiliza-se a análise de cluster, primeiro com base nas variáveis originais e, posteriormente, com os escores fatoriais, para geração de grupos que retratem as características 
comuns encontradas na análise fatorial. As variáveis e fatores resultantes são então utilizados na análise discriminante, para corroborar os resultados obtidos.

\section{Resultados da análise fatorial}

A primeira parte da análise refere-se à verificação da adequação do uso da análise fatorial para os objetivos do estudo. Verificou-se que para o conjunto das variáveis estudadas, algumas apresentaram baixa correlação entre si, indicando cautela na utilização da técnica. O requisito primário da análise fatorial é que as correlações entre as variáveis sejam significativas. Do mesmo modo, espera-se que variáveis altamente correlacionadas entre si também se correlacionem com os mesmos fatores.

Buscando maior precisão nos resultados da análise fatorial, é utilizada a medida MSA - Measures of Sampling Adequacy (Medidas de Adequação Amostral) na escolha das variáveis a serem consideradas. Tal medida é obtida pelos quadrados das correlações múltiplas da diagonal da matriz de correlação anti-imagem. A matriz anti-imagem representa a matriz de correlações entre os resíduos depois de retirada a covariância. Indica-se a eliminação das variáveis cujas correlações possam afetar a obtenção dos fatores comuns. Como critério de escolha das variáveis, foi utilizada a indicação da literatura para a exclusão daquelas que apresenta MSA $<0,5$. Assim, as variáveis desconsideradas para efeito da extração dos fatores foram: Exportações/Reservas (Exp/R), Multiplicador M2 (M2mult), Termos de Troca (TT), PIB e Taxa Real de Juros $\left(\mathrm{i}_{\mathrm{r}}\right)$. Destaque-se que no resultado da matriz dos componentes submetidos à rotação tais variáveis possuem cargas fatoriais abaixo de $40 \%$. Dessa forma, dos 13 indicadores apurados inicialmente, cinco mostraram-se inadequados aos procedimentos a serem utilizados.

Não obstante terem sido constatadas correlações baixas entre algumas das variáveis do estudo, o teste KMO para medida de adequação da amostra à análise fatorial indica a possibilidade de utilização da metodologia, assim como o teste de esfericidade de Bartlett, conforme Tabela 1 apresentada a seguir.

Tabela 1: Testes de adequação amostral

\begin{tabular}{llr}
\hline \multicolumn{3}{c}{ Testes KMO e Bartlett } \\
\hline Medida de Adequação de Kaiser-Meyer & & 0,5120 \\
& Aprox. Qui Quadrado & 642,4850 \\
& Graus de liberdade (gl) & 28 \\
Teste de Esfericidade de Bartlett & Significância & $<0,001$ \\
& & \\
\hline Elaborada pelos autores & &
\end{tabular}

Fonte: Elaborada pelos autores 
Segundo Hair et al. (1998), valores de KMO acima de 0,50 para cada variável ou para a matriz completa permitem a aplicação da análise fatorial de forma apropriada. A esfericidade de Bartlett leva à não aceitação da hipótese de correlação nula entre as variáveis, indicando que essas são significativamente correlacionadas.

No processo de estimação fatorial com várias variáveis, a obtenção de muitos componentes pode dificultar sua interpretação e torna-se importante um critério de retenção de fatores suficientes para explicar um percentual relevante da variância amostral. O processo de escolha para o número adequado de fatores baseia-se nos seguintes critérios: i) componentes ou fatores com autovalores superiores a um; ii) variância explicada por cada fator e variância acumulada; iii) interpretação dos fatores. Além disso, assumiu-se que apenas variáveis com cargas fatoriais acima de $60 \%$ seriam selecionadas para interpretar os fatores ou componentes principais, conforme critério estabelecido por Manly (2004). A partir dos critérios indicados anteriormente, verifica-se na Tabela 2 abaixo a extração de três fatores pelo método de componentes principais com autovalores superiores a 1 e que respondem juntos por aproximadamente $74 \%$ da variância total.

Tabela 2: Extração dos Fatores

\begin{tabular}{|c|c|c|c|c|c|c|}
\hline \multicolumn{7}{|c|}{ Variância Total Explicada } \\
\hline \multirow[b]{2}{*}{ Componentes } & \multicolumn{3}{|c|}{ Autovalores Iniciais } & \multicolumn{3}{|c|}{$\begin{array}{c}\text { Soma de Quadrados de Cargas } \\
\text { Rotadas }\end{array}$} \\
\hline & Total & $\begin{array}{c}\% \text { da } \\
\text { Variância }\end{array}$ & $\begin{array}{c}\% \\
\text { Acumulada }\end{array}$ & Total & $\begin{array}{c}\% \\
\text { da Variância }\end{array}$ & $\begin{array}{c}\% \\
\text { Acumulada }\end{array}$ \\
\hline 1 & 2,541 & 31,768 & 31,768 & 2,172 & 27,151 & 27,151 \\
\hline 2 & 1,822 & 22,775 & 54,542 & 2,087 & 26,084 & 53,235 \\
\hline 3 & 1,546 & 19,327 & 73,870 & 1,651 & 20,634 & 73,870 \\
\hline 4 & 0,816 & 10,203 & 84,072 & & & \\
\hline 5 & 0,540 & 6,755 & 90,827 & & & \\
\hline 6 & 0,452 & 5,653 & 96,480 & & & \\
\hline 7 & 0,212 & 2,649 & 99,129 & & & \\
\hline 8 & 0,070 & 0,871 & 100,00 & & & \\
\hline
\end{tabular}

Fonte: Elaborada pelos autores

A Tabela 3 a seguir apresenta a matriz dos escores fatoriais, relativos a cada variável sob estudo, obtida com a rotação dos componentes. Conforme explicado no referencial teórico, a rotação se faz necessária para obtenção de fatores não correlacionados entre si. Neste trabalho utiliza-se o método de extração Varimax, que origina fatores ortogonais. A especificação dos indicadores apresentados encontra-se no Apêndice. 


\begin{tabular}{cccc}
\hline & \multicolumn{3}{c}{ Fatores } \\
\hline Variáveis & 1 & 2 & 3 \\
DepBan/PIB & 0,963 & $-0,005$ & 0,050 \\
CredDom/PIB & 0,913 & $-0,009$ & 0,118 \\
Def/PIB & 0,604 & 0,265 & $-0,002$ \\
Imp/R & 0,167 & 0,864 & 0,036 \\
M2/R & $-0,011$ & 0,829 & 0,320 \\
$R$ & $-0,073$ & $-0,762$ & 0,309 \\
Er & $-0,026$ & 0,014 & $-0,872$ \\
$p$ & 0,108 & 0,045 & 0,822 \\
\hline
\end{tabular}

Notas: Extração dos fatores por Análise de Componentes Principais (Método de Rotação Varimax). Fonte: Elaborada pelos autores

A partir dos escores fatoriais calculados, de acordo com a tabela acima, e considerando-se os sinais esperados das variáveis, verifica-se a concentração no primeiro fator, com as maiores cargas fatoriais, de variáveis relacionadas à expansão do crédito doméstico, déficits governamentais crescentes e à elevação da razão de depósitos bancários em relação ao PIB. Isso está representado pelas variáveis CredDom/PIB (crédito doméstico/PIB), Def/PIB (déficit fiscal/PIB) e DepBan/PIB (depósitos bancários de não residentes/PIB). Os dois primeiros indicadores desse fator estão fortemente relacionados a problemas nos fundamentos da economia e o último retrata fragilidades de natureza financeira.

No segundo componente extraído, estão variáveis que capturam as perdas nas reservas internacionais de um país diante de uma crise cambial. Isso está retratado no crescimento das razões de importações e oferta monetária em relação às reservas (Imp/R e M2/R), bem como na queda propriamente dita do volume total dessas reservas. Tais fatores indicam fragilidade no balanço de pagamentos, destacando-se que o indicador $\mathrm{M} 2 / \mathrm{R}$ também se relaciona a problemas financeiros que levam ao chamado "comportamento de manada".

Para o último fator, os indicadores que possuem as maiores cargas estão relacionados à sobrevalorização da moeda doméstica que antecede as crises cambiais e aos níveis de produção industrial. As variáveis $\mathrm{e}_{\mathrm{r}}$ (taxa real de câmbio) e P (índice de produção industrial) retratam essa condição. Confirma-se a apreciação cambial que precede as crises. Entretanto, percebe-se o sinal positivo para o indicador de produção industrial, indicando que as crises são também precedidas por expansão econômica, como no caso dos países do Leste Asiático.

Voltando ao objetivo principal de determinar fatores comuns às crises, independentemente de suas naturezas, vale destacar a relação dos resultados obtidos com os modelos de crises cambiais existentes na literatura.

Resumidamente, os modelos de primeira geração têm foco na deterioração dos fundamentos das economias. Nesses casos, são motivos de preocupação os déficits fiscais sendo financiados monetariamente, a expansão do crédito doméstico e per- 
das persistentes de reservas internacionais, além do volume de reservas que pode ser utilizado para custear alguns meses de importações. Nos três fatores encontrados, observam-se indicadores que capturam essas fragilidades, o que pode ser constatado pelos escores das variáveis Def/PIB (déficit fiscal/PIB), CredDom/PIB (crédito doméstico/PIB), M2/R (oferta de moeda e quase-moeda/reservas), R (volume de reservas), Imp/R (importações/reservas) e $e_{r}$ (taxa real de câmbio).

No caso dos modelos de crises cambiais de segunda geração, o foco está no dilema do governo em relação ao custo-benefício da manutenção do regime de câmbio fixo. Sob um regime taxas fixas, o benefício de reduzir a inflação pode ser ultrapassado pelo custo que as altas taxas de juros terão em termos de desemprego, dificuldades para financiar o déficit público ou de maior vulnerabilidade de um setor bancário enfraquecido. Essa manutenção tem consequências também na liquidez da economia. Destaque-se que a taxa real de juros e o indicador de liquidez que relaciona a oferta de moeda e quase moeda com a base monetária (M2mult) não apresentam um carga fatorial significativa, reforçando o motivo de sua retirada das análises.

Os modelos de terceira geração dão ênfase aos problemas de contágio das crises cambiais decorrentes de fragilidades nos sistemas bancários dos países envolvidos, nos moldes da crise do Leste Asiático em 1997-1998. Nesse contexto, as variáveis relacionadas ao setor financeiro da economia e às consequências das quebras no sistema bancário dos países são as mais utilizadas na literatura. No caso do presente estudo, o primeiro componente extraído na análise fatorial contempla alguns desses indicadores de crises cambiais: CredDom/PIB (crédito doméstico/PIB), DepBan/PIB (depósitos bancários de não residentes/PIB) e M2/R (oferta de moeda e quase moeda/reservas).

Portanto, pelos resultados obtidos na análise fatorial, constata-se a existência de fatores comuns que determinam crises cambiais de naturezas diferentes e referenciadas na literatura econômica por indicadores relacionados a diferentes gerações de modelos de crises.

As variáveis representativas em cada fator extraído (com cargas fatoriais superiores a $60 \%$ ) estão relacionadas tanto à deterioração dos fundamentos quanto a problemas no balanço de pagamentos e fragilidades financeiras, independentemente de sua classificação em tipos determinados de crises, a época de suas ocorrências e respectivas gerações de modelos a elas associados. Assim, da mesma forma que Moreira, Pinto e Souza (2004), no estudo de países asiáticos, é possível determinar uma relação de interdependência entre as variáveis, expressas por fatores comuns, sem que haja, necessariamente, a condição de existência de uma relação teórica entre elas.

Tal fato é reforçado por Kaminsky e Reinhart (1999), ao lembrar que uma fonte de não linearidade impossível de capturar num modelo padrão de regressão é que a crise torna-se mais provável quando o número de fragilidades aumenta. Como exemplos, citam uma apreciação real do câmbio em certa magnitude tornando-se mais perigosa se combinada a uma excessiva expansão monetária. Portanto, salienta-se que o presente trabalho, em tal contexto, pela utilização do mé- 
todo de análise discriminante, pode identificar essa correlação entre as variáveis (apreciação da taxa de câmbio e excessiva expansão monetária ou dívida externa e altas taxas de juros) em forma de fatores, permitindo agregar um maior número de fragilidades que poderiam antecipar melhor uma crise.

O próximo passo é a confirmação desses resultados pela utilização de análises de classificação como a análise de conglomerados e a análise discriminante. Investiga-se a existência de grupos homogêneos, com valores semelhantes dos escores produzidos pela análise fatorial. Utiliza-se também a base de dados formada pelos indicadores para determinar o número de conglomerados. Os resultados são muito similares à análise de clusters com base nos escores fatoriais. A adequação do estabelecimento desses grupos será verificada no primeiro momento pela análise de cluster e, em seguida, pela utilização da função discriminante de Fischer, conforme descrito em Johnson e Wichern (1992), como uma análise confirmatória.

A partir da determinação dos grupos com base na análise de agrupamentos, conforme explicitado no parágrafo anterior, pode-se testar o poder discriminatório dos escores fatoriais contra os grupos previamente definidos. Dessa forma, os escores fatoriais obtidos são usados como variáveis independentes tanto para explicar as diferenças entre os clusters formados quanto na verificação de seu poder discriminante para a diferenciação dos grupos.

\section{Resultados da análise de agrupamentos}

A análise de agrupamentos neste trabalho é usada em duas etapas, com o propósito de confirmar a existência de grupos distintos de variáveis com características comuns dentro do mesmo agrupamento e a grande diferenciação entre um e outro grupo.

$\mathrm{Na}$ primeira etapa, parte-se dos mesmos indicadores utilizados na obtenção dos fatores da seção anterior. Pretende-se verificar se as variáveis econômicas estudadas possibilitam separar e identificar os grupos cujos elementos possuam características semelhantes entre si e diferenciadas entre os grupos. Assim, tenta-se encontrar uma relação entre os países envolvidos nos episódios de crise sob análise de acordo com o comportamento de seus indicadores econômico-financeiros naquelas épocas.

No passo seguinte, utilizam-se os escores fatoriais obtidos na seção anterior como variáveis independentes para explicar a diferenciação dos clusters encontrados e verificar seu poder de discriminar os grupos.

Como visto na quarta seção, a escolha do número de clusters a ser considerado na análise é um passo importante na interpretação correta das características dos grupos encontrados. Neste trabalho, utiliza-se o coeficiente de correlação múltipla $R^{2}$ entre grupos como critério de escolha do número de clusters a ser utilizado. A variação dos quadrados das distâncias euclidianas a cada etapa da aglomeração também é analisada para corroborar o número de clusters indicado pelo critério do $R^{2}$. 
Aplicando-se o método hierárquico aglomerativo de Ward, verificou-se a existência de dois grupos diferenciados. A confirmação de que tal separação é apropriada é feita com base no critério citado acima e seus resultados estão apresentados na Tabela 4 a seguir:

Tabela 4: $\mathrm{N}^{\circ}$ de Clusters - Critério $\mathrm{R}^{2}$

\begin{tabular}{ccc}
\hline & Análise de Agrupamento & \\
\hline No. de Clusters & $R 2$ & 0 \\
1 & 2 & 0,202 \\
3 & 0,349 \\
4 & 0,475 \\
5 & 0,611 \\
6 & 0,562 \\
7 & 0,611 \\
8 & 0,658 \\
9 & 0,692 \\
10 & 0,740 \\
\hline
\end{tabular}

Fonte: Elaborada pelos autores. Nota: $\mathrm{R}^{2}$ = quadrado da correlação múltipla.

O coeficiente $R^{2}$ fornece uma medida do percentual da variabilidade total que é retida em cada uma das soluções dos agrupamentos. A transição de $k+1$ para $k$ grupos acontece quando há um decréscimo significativo do coeficiente.

A Tabela 4 mostra um forte decréscimo do $R^{2}$ do $3^{\circ}$ para o $2^{\circ}$ grupo, passando de 0,349 para 0,202 , o que representa uma queda superior a $70 \%$. Ao passar-se do cluster 4 para o 3, a queda é da ordem de 36\%. São apresentados os cálculos para $R^{2}$ considerando apenas os dez últimos agrupamentos, por não ser necessário observar as variações do coeficiente em todo o processo de aglomeração. A análise das variações da distância euclidiana a cada aglomeração confirma a decisão pelo critério do coeficiente de correlação múltipla. A análise do dendograma, por sua vez, confirma a opção de dois grupos. Portanto, conclui-se pela escolha de dois clusters.

Os clusters encontrados estão detalhados nas Tabelas 5 e 6 e apresentados a seguir. A análise dos elementos dos grupos é um passo inicial na identificação de suas particularidades. A título de referência, foi incluída na tabela, para cada país, a classificação de crises cambiais desenvolvida por Kaminsky (2003). 
Tabela 5: Agrupamentos Obtidos: Grupo 1

\begin{tabular}{|c|c|c|}
\hline & Grupo 1 & \\
\hline País & Data da Crise & Tipo de Crise (1) \\
\hline Argentina & Jun./70 & Não classificada \\
\hline Argentina & Fev./81 & Financeira \\
\hline Argentina & Jul./82 & Financeira \\
\hline Bolívia & Fev./83 & Financeira \\
\hline Chile & Ago./72 & Dívida soberana \\
\hline Chile & Out./73 & Déficit fiscal \\
\hline Colômbia & Fev./85 & Financeira \\
\hline Filipinas & Out./83 & Financeira \\
\hline Filipinas & Jun./84 & Financeira \\
\hline Filipinas & Fev./86 & Dívida soberana \\
\hline Indonésia & Abr./83 & Financeira \\
\hline Israel & Jul./84 & Dívida soberana \\
\hline México & Set./76 & Dívida soberana \\
\hline México & Dez./94 & Financeira \\
\hline Peru & Jun./76 & Conta corrente \\
\hline Peru & Out./87 & Dívida soberana \\
\hline Peru & Set./88 & Dívida soberana \\
\hline Tailândia & Jul./81 & Conta corrente \\
\hline Tailândia & Nov./84 & Financeira \\
\hline Uruguai & Dez./71 & Dívida soberana \\
\hline Uruguai & Out./82 & Financeira \\
\hline
\end{tabular}

Nota: (1) Classificação dada por Kaminsky (2003). Fonte: Elaborada pelos autores

Tabela 6: Agrupamentos Obtidos: Grupo 2

\begin{tabular}{|c|c|c|}
\hline \multicolumn{2}{|c|}{ Grupo 2 } \\
\hline País & Data da Crise & Tipo de Crise (1) \\
\hline Argentina & Jun./75 & Financeira \\
\hline Argentina & Set./86 & Dívida soberana \\
\hline Argentina & Fev./02 & Conta corrente \\
\hline Bolívia & Nov./82 & Financeira \\
\hline Bolívia & Nov./83 & Financeira \\
\hline Brasil & Fev./83 & Dívida soberana \\
\hline
\end{tabular}

continua 


\begin{tabular}{|c|c|c|}
\hline Brasil & Nov./86 & Divida soberana \\
\hline Brasil & Jul./89 & Dívida soberana \\
\hline Brasil & Out./91 & Dívida soberana \\
\hline Brasil & Jan./99 & Financeira \\
\hline Chile & Dez./71 & Dívida soberana \\
\hline Chile & Ago./82 & Dívida soberana \\
\hline Chile & Set./84 & Dívida soberana \\
\hline Colômbia & Mar./83 & Financeira \\
\hline Colômbia & Ago./95 & Financeira \\
\hline Colômbia & Set./97 & Conta corrente \\
\hline Colômbia & Set./98 & Financeira \\
\hline Colômbia & Ago./99 & Financeira \\
\hline Dinamarca & Mai./71 & Autorrealizável \\
\hline Dinamarca & Jun./73 & Autorrealizável \\
\hline Dinamarca & Nov./79 & Conta corrente \\
\hline Dinamarca & Ago./93 & Dívida soberana \\
\hline Espanha & Fev./76 & Conta corrente \\
\hline Espanha & Jul./77 & Conta corrente \\
\hline Espanha & Dez./82 & Dívida soberana \\
\hline Espanha & Set./92 & Financeira \\
\hline Espanha & Mai./93 & Financeira \\
\hline Filipinas & Fev./70 & Não classificada \\
\hline Filipinas & Dez./97 & Dívida soberana \\
\hline Finlândia & Jun./73 & Autorrealizável \\
\hline Finlândia & Out./82 & Sudden stops \\
\hline Finlândia & Nov./91 & Dívida soberana \\
\hline Finlândia & Set./92 & Dívida soberana \\
\hline Indonésia & Nov./78 & Conta corrente \\
\hline Indonésia & Set./86 & Dívida soberana \\
\hline Indonésia & Dez./97 & Dívida soberana \\
\hline Indonésia & Jan./98 & Dívida soberana \\
\hline Israel & Nov./74 & Conta corrente \\
\hline Israel & Nov./77 & Financeira \\
\hline Israel & Out./83 & Dívida soberana \\
\hline Malásia & Jul./75 & Conta corrente \\
\hline
\end{tabular}




\begin{tabular}{|c|c|c|}
\hline Malásia & Ago./97 & Financeira \\
\hline Malásia & Jun./98 & Financeira \\
\hline México & Fev./82 & Financeira \\
\hline México & Dez./94 & Financeira \\
\hline Noruega & Jun./73 & Autorrealizável \\
\hline Noruega & Fev./78 & Financeira \\
\hline Noruega & Mai./86 & Sudden stops \\
\hline Noruega & Dez./92 & Déficit fiscal \\
\hline Noruega & Jul./98 & Dívida soberana \\
\hline Noruega & Jul./99 & Dívida soberana \\
\hline Noruega & Nov./00 & Dívida soberana \\
\hline Suécia & Ago./77 & Conta corrente \\
\hline Suécia & Set./81 & Sudden stops \\
\hline Suécia & Out./82 & Sudden stops \\
\hline Suécia & Nov./92 & Dívida soberana \\
\hline Tailândia & Nov./78 & Conta corrente \\
\hline Tailândia & Jul./97 & Dívida soberana \\
\hline Tailândia & Set./99 & Dívida soberana \\
\hline Tailândia & Jul./00 & Déficit fiscal \\
\hline Turquia & Ago./70 & Não classificada \\
\hline Turquia & Fev./01 & Financeira \\
\hline Venezuela & Fev./84 & Financeira \\
\hline Venezuela & Dez./86 & Dívida soberana \\
\hline Venezuela & Mar./89 & Dívida soberana \\
\hline
\end{tabular}

Nota: (1) Classificação dada por Kaminsky (2003). Fonte: Elaborada pelos autores

Os oito indicadores utilizados na obtenção dos fatores na análise fatorial possibilitaram separar em dois grupos os países e as respectivas crises por eles enfrentados.

Percebe-se que o Grupo 1 comporta a maior parte das crises ocorridas antes da década de 1990. O Grupo 2, mesmo contendo episódios de crise da mesma época do primeiro agrupamento, é composto, predominantemente, por crises ocorridas a partir dos anos 1990, inclusive a grande onda de crises do Leste Asiático.

Outra observação importante é quanto à relação dos tipos de crise dentro dos grupos. Com o objetivo de estabelecer uma primeira relação entre os grupos formados e as diferentes naturezas das crises, foi incluída a classificação estabelecida por Kaminsky (2003) como referência.

Verifica-se que não é possível observar diretamente uma caracterização clara 
entre os grupos estabelecidos com base nos tipos de crise. O resultado da composição dos clusters reforça a ideia de existência de características comuns às crises que independem de sua classificação.

Assim, tenta-se encontrar uma classificação entre os países envolvidos nos episódios de crise sob análise de acordo com o comportamento dos indicadores econômico-financeiros relacionados a tais episódios.

Partindo-se dos dois grupamentos identificados na análise de clusters, utiliza-se a análise discriminante para confirmar se os grupos são significativamente distintos entre si.

\section{Resultados da análise discriminante}

Com a obtenção dos dois grupos na análise anterior, utiliza-se a análise discriminante para confirmar a diferenciação entre os grupos evidenciados. As variáveis que diferenciam-se significativamente entre clusters podem ser identificadas por meio dessa técnica.

A primeira análise é feita com base nas variáveis utilizadas para a formação dos grupos da seção anterior. Em outro momento, faz-se a mesma análise a partir dos escores fatoriais obtidos na parte inicial dos resultados deste trabalho.

Para a seleção das variáveis discriminantes, utiliza-se o teste de igualdade de médias dos grupos, verificando-se suas significâncias, conforme indicado na Tabela 7 abaixo:

Tabela 7: Teste de Significância

\begin{tabular}{cccc}
\hline & \multicolumn{3}{c}{ Teste de Igualdade de Médias dos Grupos } \\
\hline Variáveis & Lambda de Wilks & $\mathrm{F}$ & Sig. \\
\hline DepBan/PIB & 0,947 & 9,161 & 0,002 \\
CredDom/PIB & 0,902 & 17,699 & $<0,000$ \\
Def/PIB & 0,916 & 14,990 & $<0,000$ \\
Imp/R & 0,573 & 122,095 & $<0,000$ \\
M2/R & 0,534 & 143,035 & $<0,000$ \\
Er & 0,969 & 5,181 & 0,024 \\
$P$ & 0,827 & 34,171 & $<0,000$ \\
R & 0,861 & 26,270 & $<0,000$ \\
\hline
\end{tabular}

Nota: Graus de liberdade $(1,164)$. Fonte: Elaborada pelos autores

Verifica-se que, ao nível de significância $\alpha$ de 0,05 , todas se mostram com poder de discriminação. Isso era esperado em função da eliminação prévia, na análise fatorial, das variáveis não apropriadas.

Com base na análise das médias da Tabela 8, constata-se que o Grupo 1 é o mais vulnerável com base nos oito indicadores utilizados, exceto pelo indicador Depósitos Bancários/PIB. 
Tabela 8: Análise das Médias dos Grupos

\begin{tabular}{ccccc}
\hline \multicolumn{5}{c}{ Estatísticas de Grupos } \\
\hline Grupos & Variáveis & Médias & Desvio-Padrão & Coeficiente de \\
\hline 1 & DepBan/PIB & $-0,0009$ & 0,464 & $-515,55$ \\
1 & CredDom/PIB & 0,128 & 0,587 & 4,58 \\
1 & Def/PIB & 0,066 & 0,470 & 7,12 \\
1 & Imp/R & 0,776 & 1,022 & 1,31 \\
1 & M2/R & 0,967 & 1,123 & 1,16 \\
1 & Er & $-0,241$ & 0,332 & $-1,37$ \\
1 & $\mathrm{P}$ & 0,580 & 1,041 & 1,79 \\
2 & $\mathrm{R}$ & $-0,505$ & 0,613 & $-1,21$ \\
2 & DepBan/PIB & $-0,204$ & 0,378 & $-1,85$ \\
2 & CredDom/PIB & $-0,232$ & 0,487 & $-2,09$ \\
2 & Def/PIB & $-0,224$ & 0,450 & $-2,00$ \\
2 & Imp/R & $-0,449$ & 0,398 & $-0,88$ \\
2 & M2/R & $-0,480$ & 0,426 & $-0,88$ \\
2 & Er & 0,132 & 1,204 & 9,12 \\
2 & $\mathrm{P}$ & $-0,303$ & 0,852 & -2.81 \\
\hline
\end{tabular}

Fonte: Elaborada pelos autores

A análise discriminante origina uma função que tenha significância para explicar a variância entre os grupos. Nessa análise, os grupos são representados pela nova variável categórica dependente formada pelos clusters 1 e 2 , a partir da análise de conglomerados. A Tabela 9 apresenta os resultados das estatísticas relevantes para analisar o poder discriminante da função encontrada.

Tabela 9: Estatísticas Relacionadas à Função Discriminante

\begin{tabular}{ccccc}
\hline \multicolumn{5}{c}{ Autovalores } \\
\hline Função & Autovalor & \% da Variância & \% Acumulado & $\begin{array}{c}\text { Correlação } \\
\text { Canônica } \\
1\end{array}$ \\
\hline & 1.826 & 100 & 100 & 0,804 \\
\hline Teste da Função & Lambda de Wilks & Qui quadrado & Gl & Sig \\
1 & 0,353 & 166,252 & 8 & $<0,000$ \\
\hline
\end{tabular}

Fonte: Elaborada pelos autores 
Pelas estatísticas acima, constata-se que a função tem poder para discriminar os Grupos 1 e 2. Tomando-se o quadrado da correlação canônica, verifica-se que aproximadamente $65 \%$ da variância dos clusters é explicada pela função obtida. Quanto ao lambda de Wilks, verifica-se a significância da função na diferenciação dos grupos.

O poder de classificação da função pode ser verificado na Tabela 10 ao apresentar um alto poder de predição, ou seja, $97,6 \%$ dos casos de cada grupo são corretamente classificados.

Tabela 10: Estatística de Classificação da Função Discriminante

\begin{tabular}{ccccc}
\hline \multicolumn{5}{c}{ Resultados da Classificação ${ }^{*}$ ) } \\
\hline Método Ward & \multicolumn{1}{c}{ N $^{\circ}$ de Países Preditos } & Total \\
\hline Contagem original & Grupo 1 & 53 & Grupo 2 & \\
Contagem original & Grupo 2 & 1 & 3 & 56 \\
$\%$ & Grupo 1 & 94,643 & 109 & 110 \\
$\%$ & Grupo 2 & 0,909 & 5,357 & 100 \\
\hline
\end{tabular}

Nota: $\left({ }^{*}\right)$ 97,6\% dos casos de cada grupo foram classificados corretamente. Fonte: Elaborada pelos autores

Faz-se relevante, ainda, analisar a função discriminante com base nos escores da análise fatorial, como técnica confirmatória da diferenciação dos dois grupos encontrados na análise de conglomerados.

Utilizando-se como variáveis independentes os escores dos três fatores obtidos e como variável dependente a nova variável categórica resultante da análise de clusters, busca-se obter a função que melhor discrimine os Grupos 1 e 2 encontrados.

Os resultados das principais estatísticas para verificação do poder discriminante da função encontrada são relacionados abaixo, nas Tabelas 11, 12 e 13.

Tabela 11: Teste de Significância para Médias

\begin{tabular}{cccc}
\hline & \multicolumn{1}{c}{ Testes de Igualdade das Médias dos Grupos } & \\
\hline Variáveis & Lambda de Wilks & $\mathrm{F}$ & Sig. \\
Fator 1 & 0,960 & 6,747 & 0,010 \\
Fator 2 & 0,545 & 136,867 & $<0,000$ \\
Fator 3 & 0,883 & 21,799 & $<0,000$ \\
\hline
\end{tabular}

Fonte: Elaborada pelos autores. Graus de liberdade $(1,164)$ 


\begin{tabular}{cccc}
\hline \multicolumn{4}{c}{ Estatísticas dos Grupos } \\
\hline Grupos & Variáveis & Médias & Desvio-padrão \\
1 & Fator 1 & $-0,0009$ & 0,464 \\
1 & Fator 2 & 0,128 & 0,587 \\
1 & Fator 3 & 0,066 & 0,470 \\
2 & Fator 1 & $-0,204$ & 0,378 \\
2 & Fator 2 & $-0,233$ & 0,487 \\
2 & Fator 3 & $-0,224$ & 0,450 \\
\hline
\end{tabular}

Fonte: Elaborada pelos autores

Tabela 13: Estatísticas Relacionadas à Função Discriminante

\begin{tabular}{|c|c|c|c|c|}
\hline \multicolumn{5}{|c|}{ Autovalores } \\
\hline Função & Autovalor & \% da Variância & $\%$ Acumulado & $\begin{array}{c}\text { Correlação } \\
\text { Canônica }\end{array}$ \\
\hline 1 & 1,467 & 100 & 100 & 0,771 \\
\hline \multicolumn{5}{|c|}{ Lambda de Wilks } \\
\hline Teste da Função & Lambda de Wilks & Qui quadrado & $\mathrm{Gl}$ & Sig \\
\hline 1 & 0,405 & 146,775 & 3 & $<0,000$ \\
\hline
\end{tabular}

Fonte: Elaborada pelos autores

Pelos resultados da Tabela 11, verifica-se que os três fatores obtidos são significativos para diferenciação dos Grupos 1 e 2, considerando-se um nível de significância $\alpha$ igual a $5 \%$.

$\mathrm{Na}$ Tabela 12, pela análise das médias dos grupos em relação a seus valores e sinais esperados, constata-se que o Grupo 1 possui as maiores médias para o Fator 1, que representa os indicadores Depósitos Bancários/PIB, Crédito Doméstico/PIB e Déficit Público/PIB; para o Fator 2, que reflete as variações dos indicadores Importações/Reservas, M2/Reservas e Reservas; e também para o Fator 3, que agrega com maior significância as variáveis Taxa Real de Câmbio e Índice de Produção Industrial. Tais resultados corroboram a análise anterior, quanto à definição do cluster 1 como o possuidor de maiores características de vulnerabilidade.

A Tabela 13 traz os coeficientes que indicam o poder discriminatório da função encontrada. O coeficiente de correlação canônica indica que a função obtida a partir dos escores fatoriais tem grande poder de explicação da variância entre os Grupos 1 e 2. Já o teste relacionado ao lambda de Wilks mostra que a função é significativa na diferenciação dos grupos. 
O poder classificatório da função é confirmado pelos resultados apresentados na Tabela 14 abaixo. Os dados apresentam uma capacidade de predição de aproximadamente $97 \%$, ou seja, $97 \%$ dos casos de cada grupo são classificados corretamente com o uso da função obtida.

Tabela 14: Estatísticas de Classificação da Função Discriminante

\begin{tabular}{|c|c|c|c|c|}
\hline \multicolumn{5}{|c|}{ Resultados da Classificação $\left({ }^{*}\right)$} \\
\hline \multicolumn{2}{|c|}{ Método Ward } & \multicolumn{2}{|c|}{$N^{\circ}$ de Países Preditos } & \multirow[t]{2}{*}{ Total } \\
\hline & & Grupo 1 & Grupo 2 & \\
\hline Contagem original & Grupo 1 & 51 & 5 & 56 \\
\hline Contagem original & Grupo 2 & 0 & 110 & 110 \\
\hline$\%$ & Grupo 1 & 91,071 & 8,929 & 100 \\
\hline$\%$ & Grupo 2 & 0 & 100 & 100 \\
\hline
\end{tabular}

Nota: $\left({ }^{*}\right) 97,0 \%$ dos casos de cada grupo foram classificados corretamente. Fonte: Elaborada pelos autores

\section{CONCLUSÕES}

A utilização de técnicas estatísticas de análise multivariada, em especial da análise fatorial, de conglomerados e discriminante, permitiu a identificação e avaliação de fatores comuns determinantes de crises cambiais ocorridas no período entre 1970 e 2004, que envolveram tanto economias maduras, quanto os chamados países emergentes.

Os resultados obtidos confirmaram a existência de oito indicadores econômico-financeiros, frequentemente citados na literatura, comuns a crises cambiais de diferentes naturezas e épocas. Constatou-se que a elevação das proporções Crédito Doméstico/PIB, Déficit Fiscal/PIB e Depósitos Bancários/PIB são inerentes à diversidade de crises classificada pela literatura econômica.

Além disso, foram identificados como fatores comuns aos episódios indicadores que capturam a excessiva monetização das economias e refletem a queda das reservas dos bancos centrais. Foram eles: M2/Reservas, Importações/Reservas e volume total das reservas internacionais dos países. A taxa real de câmbio e o índice de produção industrial também se mostraram comuns aos episódios de crises estudados.

Com base nos fatores identificados, foi possível classificar os países em dois grupos significativamente distintos. O primeiro deles, concentrando a maior parte das crises ocorridas nas décadas de 1970 e 1980, registra maior incidência de problemas de origem financeira, representando $48 \%$ do total de episódios, contra $28 \%$ do Grupo 2. Por sua vez, o segundo grupamento de países, que reflete principalmente os problemas cambiais a partir dos anos 1990 , tem predominância de crises relacionadas a divida soberana, na proporção de $38 \%$ do total de casos estudados.

Cabe ressaltar que os trabalhos empíricos, em geral, têm como foco a previsão 
de crises cambiais e financeiras e a identificação das diferentes naturezas ou causas de tais episódios de crise. Neste artigo procuramos responder outra pergunta. $\mathrm{O}$ que há em comum entre os diversos episódios de crises? Nesse contexto, utilizamos a análise fatorial para revelar os indicadores de crise comuns a todos os eventos analisados e, posteriormente, utilizamos a análise de classificação (análise de agrupamento e análise discriminante) para avaliar com base nos escores dos fatores comuns se há distinção de grupos de países por episódio de crise. A investigação sobre os fatores comuns às crises e a metodologia aqui adotada ainda não tinham sido empregados na literatura internacional para essa literatura.

\section{REFERÊNCIAS BIBLIOGRÁFICAS}

EICHENGREEN, B., ROSER, A. e WYPLOSZ, C. (1996), "Exchange market mayhem: the antecedents and aftermath of speculative attacks", Economic Policy, Oct., pp. 249-312.

FRANKEL, J. e ROSE, A. (1996), "Currency Crashes in Emerging Markets: Empirical Indicators", NBER Working Paper Series, Working Paper 5437, Cambridge, MA: National Bureau of Economic Research, Jan.

HAIR, Joseph F.; TATHAM Ronald L.; ANDERSON, Rolph E.; BLACK, William, (1998), "Multivariate data analysis", 5th edition, New Jersey: Prentice Hall,.

JOHNSON,R.A.e WICHERN,D. W. (1992), “Applied multivariate statistical analysis”, 3th edition, New Jersey: Prentice Hall,.

KAMINSKY, G.L., (2003) “Varieties of Currency Crises”, NBER Working Paper No. 10193, December.

KAMINSKY e REINHART, C.M. (1999), "The Twin Crises: The Causes of Banking and Balanceof-Payments Problems," American Economic Review, June.

KAMINSKY, LIZONDO e REINHART (1997) “Leading Indicators of Currency Crises”, IMF Working Paper, WP/97/79, July.

MANLY, B. F. J. (2004), "Multivariate Statistical Methods: a Primer”, 3ª Edição, Chapman \& Hall/ CRC.

MAROCO, J. (2003), “Análise estatística com utilização do SPSS”, 2ª Edição, Lisboa: Silabo, pp. 56-59, 112-114 e 332-360.

MOREIRA, T. B. S., PINTO, M. B. de P., SOUZA, G. S. (2004), "Uma metodologia alternativa para mensuração de pressão sobre o mercado de câmbio", Estudos Econômicos, v. 34, n. 1, p. 73-100, jan./mar.

NOHARA, JOULIANA J. (1997), "Crise Financeira: Antiga e Novas Abordagens”, Revista de Economia Política, Vol. 17, n 1 (65), jan-mar.

SPEARMAN, C. (1904). "General Intelligence objectively determined and measured", American Journal of Psychology, 15, pp. 201-293.

\section{APÊNDICE - INDICADORES E FONTES}

Prioritariamente, foram utilizados dados do International Financial Statistics (IFS), International Monetary Fund (IMF). Para completar as séries de dados, foram utilizadas as seguintes publicações, seguindo a metodologia de cálculo indicada pelo IMF: Emerging Market Indicators, International Finance Corporation (IFC); European Central Bank; International Banking and Financial Market Development, Bank for International Settlements (BIS); European Statistics on line, European Commission. 
Foram, ainda, obtidos dados a partir de boletins dos bancos centrais dos países para os quais não havia disponibilidade de informações nas publicações citadas.

\section{Indicadores utilizados}

Multiplicador M2 (M2mult) - razão entre oferta de moeda e quase-moeda e a base monetária. IFS linhas 34 e 35 (M2) e linha 14 (base money); Crédito Doméstico/PIB (CredDom/PIB) — proporção do crédito doméstico em termos reais (deflacionado por índice de preços ao consumidor) em relação ao PIB. IFS linha 52 dividida por linha 64 (consumer prices), dividida pelo PIB em termos reais, IFS linha 99b.p (GDP); Depósitos Bancários/PIB (DepBan/PIB) - depósitos bancários de residentes, IFS linhas 24 (demand deposits) e 25 (time and saving deposits), deflacionados pela linha 64 (consumer prices), divididos por IFS linha 99b.p;Déficit Fiscal/PIB (Def/PIB) — razão entre o déficit ou superávit do governo, deflacionado, e o PIB. IFS linha 80 (déficit (-) or surplus), dividida pela linha 64 (consumer prices) e dividida por IFS linha 99b.p; PIB (PIB) - produto interno bruto, a preços constantes. IFS linha 99b.p (GDP); Reservas Internacionais $(\mathrm{R})$ - reservas internacionais, em dólares, IFS linha 1l.d (reserves); $\mathrm{M} 2 /$ Reservas (M2/R) — oferta de moeda, convertida em dólar, em relação às reservas internacionais. IFS linhas 34 e 35 (M2), convertido em dólar pela linha ae e dividido pela linha 1l.d (reserves); Exportações/Reservas (Exp/R) - exportações f.o.b, IFS linha 70..d (exports), dividido pela linha 1l.d (reserves); Importações/Reservas (Imp/R) - exportações f.o.b, IFS linha 71..d (imports), dividido pela linha 1l.d (reserves); Termos de Troca (TT) — valor unitário das exportações, IFS linha 74..d (export unit values) sobre valor unitário das importações IFS linha 75..d (import unit values); Produção Industrial ( $\mathrm{P})$ - índice de produção industrial, IFS linha 66 (industrial production). Para alguns países exportadores de commodities, se a produção industrial não está disponível, é usado índice de produção de produtos primários, IFS linha 66aa (crude petroleum production); Taxa Real de Câmbio $\left(\mathrm{e}_{\mathrm{r}}\right)$ - taxa de câmbio real, calculada com base no conceito de paridade relativa. Estabelecida a data de referência (2004), calcula-se as taxas de câmbio para o período $t$ a partir das taxas nominais, linha rf (official rate) e da relação entre a inflação dos Etados Unidos e do país sob análise, utilizando o índice de preços ao consumidor, linha 64 (consumer prices); Taxa Real de Juros $\left(\mathrm{i}_{\mathrm{r}}\right)$ - taxa doméstica de juros reais, em porcentual. Taxa de depósitos, IFS linha $60 \mathrm{l}$ (deposit rate), deflacionada utilizando preços ao consumidor, IFS linha 64 (consumer prices). 